\title{
ON THE CLASSIC GEOMETRODYNAMICS \\ OF A SPHERICALLY-SYMMETRIC CONFIGURATION \\ OF GRAVITATIONAL AND ELECTROMAGNETIC FIELDS
}

\author{
V.D. Gladush \\ Oles Honchar Dnipro National University, Dnipro, Ukraine \\ e-mail:vgladush@gmail.com
}

\begin{abstract}
Analytical aspects of the classical geometrodynamics for the spherically-symmetric configuration of the electromagnetic and gravitational fields in GR are considered. The feature of such configurations is that they admit two motion integrals - the total mass and charge. The Einstein-Hilbert action for the configuration, after dimensional reduction, by means of the Legendre transformation is reduced to the Hamiltonian action. Using the conservation laws for the mass and charge, as well as the Hamiltonian constraint, the momenta are found as functions of configuration variables. The set of equations, which associate momenta and functional derivatives of the action in the configuration space (CS) is integrable. This allows us to obtain the action functional as a solution of the Einstein-Hamilton-Jacobi equation in functional derivatives. Variations of the action functional with respect to mass $M$ and charge $Q$ of the configuration lead to the motion trajectories in the CS.

We note that the minisuperspace metric, which is induced by the kinetic part of the Lagrangian, does not coincide with the CS metric that arises when the function of lapse $N$ is excluded from the action. The space-time metric for which the indicated metrics coincide in the T-region up to a coefficient are considered. The metric of $\mathrm{CS}$ is constructed and its geometry is studied. Under the trivial embedding of hypersurfaces of the foliation into a dynamical T-region, the CS is flat. It allows introducing pseudoCartesian coordinates in which the CS metric takes the Lorentz form.
\end{abstract}

Keywords: spherical symmetry, electromagnetic and gravitational fields, geometrodynamics, constraints, configuration space, Einstein-Hamilton-Jacobi equation.

Received 03.09.2019; Received in revised form 10.10.2019; Accepted 15.10.2019

\section{Introduction}

As is well known, the space-time metric $\mathrm{M}^{4}$ of a spherically-symmetric configuration of the electromagnetic and gravitational fields in GR admits the Killing vector. Therefore, in the R-region, when Killing time is used, the fields do not have dynamic degrees of freedom. By virtue of this, to study quantization issues, in $[1,2]$ we limited ourselves to considering the Tregion, where these fields have a dynamic meaning. In the general geometrodynamics approach [3-5], a 3+1-splitting of $\mathrm{M}^{4}$ into a one-parameter family of the spacelike hypersurfaces is constructed. The corresponding parameter determines the time coordinate of the reference system and describes the evolution of geometric quantities given on these hypersurfaces, thereby introducing the dynamics of these objects.

In the paper [6] the classical geometrodynamics of charged black holes is considered. Herewith, uses a metric $\mathrm{M}^{4}$ of the form [3, 4]:

$$
d s^{2}=N^{2}\left(d x^{0}\right)^{2}-L^{2}\left(d r+N^{r} d x^{0}\right)^{2}-R^{2} d \sigma^{2}
$$

where $d x^{0}=c t, d \sigma^{2}=d \theta^{2}+\sin ^{2} \theta d \alpha^{2}, N$ is the function of lapse (Lagrange multiplier).

Note that the metric of minisuperspace, which is induced by the kinetic part of the Lagrangian, does not coincide with the metric of the configuration space (CS) that arises when $N$ is excluded from the action. In the T-region, they coincide up to a coefficient for metrics $\mathrm{M}^{4}$ of the form

$$
d s^{2}=n^{2} L^{-2}\left(d x^{0}\right)^{2}-L^{2}\left(d r+N^{r} d x^{0}\right)^{2}-R^{2} d \sigma^{2},
$$

where $\mathrm{n}$ is a new Lagrange multiplier. In this paper, we propose a procedure for integrating the equations of classical geometrodynamics for the system under consideration with a metric $\mathrm{M}^{4}$ in the form 


$$
d s^{2}=\frac{R}{\xi}\left(n d x^{0}\right)^{2}-\frac{\xi}{R}\left(d r+N^{r} d x^{0}\right)^{2}-R^{2} d \sigma^{2}
$$

\section{Basic dynamic quantities and constraints of the configuration}

Consider the total action for the configuration under consideration in the form

$$
S=-\frac{1}{16 \pi c} \int\left({\frac{c^{4}}{\kappa}}^{(4)} R+F_{\mu \nu} F^{\mu \nu}\right) \sqrt{-g} d x^{0} d r d \theta d \alpha+\text { (boundary terms) }
$$

where ${ }^{(4)} R$ is the Ricci scalar,

$$
F_{\mu \nu}=A_{v, \mu}-A_{\mu, \nu}
$$

is the electromagnetic field tensor, $g=\operatorname{det}\left|g_{\alpha \beta}\right|$. After integration over the angles $\theta$ and $\alpha$, the action, up to the surface terms, can be represented as

$$
\begin{gathered}
S=\int \Lambda d^{2} x, \quad \Lambda=n^{-1} T+n U, \\
T=-\frac{c^{3}}{2 \kappa}\left(\xi_{, 0}-N^{r} \xi_{, r}-2 \xi N_{, r}^{r}\right)\left(R_{, 0}-N^{r} R_{, r}\right)+\frac{1}{2 c} R^{2} E^{2}, \\
U=\frac{c^{3}}{2 \kappa}\left(1+\frac{R^{2}}{\xi^{2}} R_{, r} \xi_{, r}-\frac{2 R}{\xi} R_{, r}^{2}-\frac{2 R^{2}}{\xi} R_{, r r}\right)
\end{gathered}
$$

where $\Lambda$ is the system Lagrangian, $T$ and $U$ are the kinetic and potential parts of the Lagrangian of the system, $N$ and $N^{r}$ are lapse and shift functions, $\xi, R$, and $\phi$ are configuration variables.

Furthermore $A_{\mu}=\left\{A_{0}=\varphi, A_{r}=\phi, 0,0\right\}, F_{0 r}=-F_{r 0}=E=\phi_{, 0}-\varphi_{, r}$, $R_{, 0}=\partial R / \partial x^{0}, R_{, r}=\partial R / \partial r, \quad R_{, r r}=\partial^{2} R / \partial r^{2}$.

The canonical momenta conjugated to the configuration variables $\xi, R$, and $\phi$ are

$$
\begin{gathered}
P_{\xi}=\frac{\partial \Lambda}{\partial \xi_{, 0}}=-\frac{c^{3}}{2 \kappa n}\left(R_{, 0}-N^{r} R_{, r}\right), \\
P_{R}=\frac{\partial \Lambda}{\partial R_{, 0}}=-\frac{c^{3}}{2 \kappa n}\left(\xi_{, 0}-N^{r} \xi_{, r}-2 \xi N_{, r}^{r}\right), \\
P_{\phi}=\frac{\partial \Lambda}{\partial \phi_{, 0}}=\frac{R^{2}}{c n}\left(\phi_{, o}-\varphi_{, r}\right)=\frac{R^{2}}{c n} E .
\end{gathered}
$$

A Legendre transformation of the dynamical system gives the Hamiltonian action

$$
S=\int d x^{0} \int d r\left(P_{\xi} \xi_{, 0}+P_{R} R_{, 0}+P_{\phi} \phi_{, 0}-n H_{0}-N^{r} H_{r}-\varphi H_{\varphi}\right)
$$

where $\widetilde{H}, H_{r}$, and $H_{\varphi}$, respectively, are the Hamiltonian, diffeomorphism and Gaussian constraints

$$
H_{0}=-\frac{\delta \Lambda}{\delta n}=-\frac{2 \kappa}{c^{3}} P_{R} P_{\xi}+\frac{c}{2 R^{2}} P_{\phi}^{2}-\frac{c^{3}}{2 \kappa}\left(1+\frac{R^{2}}{\xi^{2}} R_{, r} \xi_{, r}-\frac{2 R}{\xi} R_{, r}^{2}-\frac{2 R^{2}}{\xi} R_{, r r}\right)=0,
$$




$$
\begin{gathered}
H_{r}=-\frac{\delta \Lambda}{\delta N^{r}}=R_{, r} P_{R}-\xi_{, r} P_{\xi}-2 \xi P_{\xi, r}=0, \\
H_{\varphi}=-\frac{\delta \Lambda}{\delta \varphi}=P_{\phi, r}=0 .
\end{gathered}
$$

The Gaussian constraint (13) determines the electric field strength $E$ generated by a charge $Q$

$$
P_{\phi}=\frac{R^{2}}{c n} E=\frac{Q}{c}=\text { const } \Rightarrow E=n \frac{Q}{R^{2}} .
$$

\section{The mass and momenta as functions of the configuration variables}

The Einstein equations for the configuration under consideration lead to the conservation laws for charge $Q$ and the total mass function $M_{\text {tot }}$ [7]. For the metric (2), the mass function $M_{\text {tot }}$ takes the form

$$
M_{t o t}\left(R, R_{, 0}, R_{, r}, \xi, E\right)=\frac{c^{2}}{2 \kappa}\left[R+\frac{\xi}{n^{2}}\left(R_{, 0}-N^{r} R_{, r}\right)^{2}-\frac{R^{2}}{\xi} R_{, r}^{2}\right]+\frac{R^{3}}{2 c^{2} n^{2}} E^{2}=m .
$$

Relations (7) and (14) allow us to formulate the mass in terms of momenta

$$
M_{t o t}\left(R, R_{, r}, \xi, P_{\xi}, P_{\phi}\right)=\frac{c^{2}}{2 \kappa}\left[R+\frac{4 \kappa^{2}}{c^{6}} \frac{\xi}{R} P_{\xi}^{2}-\frac{R^{2}}{\xi} R_{, r}^{2}\right]+\frac{1}{2 R} P_{\phi}^{2}=m .
$$

Hence, one can also find the momenta as functions of configuration variables. This allows us to construct the system action as a functional in the CS, which is a solution of the Einstein-Hamilton-Jacobi equation (EHJ). Indeed, from (16) we find the momentum $P_{\xi}$ as a function of $R, R_{r}$, and parameters $m$ and $Q$ :

$$
P_{\xi}=\frac{c^{3}}{2 \kappa} \sqrt{\frac{R}{\xi} F_{t o t}}, \quad F_{t o t}=\frac{R}{\xi} R_{, r}^{2}-1+\frac{2 \kappa m}{c^{2} R}-\frac{\kappa Q}{c^{4} R^{2}} .
$$

Substituting the momenta $P_{\xi}, P_{\phi}$ into the Hamiltonian constraint (11), one obtains the momentum $P_{R}$ :

$$
P_{R}=\sqrt{\frac{\xi}{R F_{\text {tot }}}}\left[\frac{Q^{2}}{2 c R^{2}}-\frac{c^{3}}{2 \kappa}\left(1+\frac{R^{2}}{\xi^{2}} R_{, r} \xi_{, r}-\frac{2 R}{\xi} R_{, r}^{2}-\frac{2 R^{2}}{\xi} R_{, r r}\right)\right]
$$

\section{The action and system trajectories in the configuration space}

The EHJ equation for the action $S$ can be obtained by substituting the functional derivatives

$$
P_{\xi}=\frac{\delta S}{\delta \xi}, \quad P_{R}=\frac{\delta S}{\delta R}, \quad P_{\phi}=\frac{\delta S}{\delta \phi}=\frac{Q}{c}
$$

into relation (11). However, instead of solving the resulting EHJ equation, we find $\mathrm{S}$ using the presented derivatives. The last equation for $P_{\phi}$ gives 


$$
S[\xi, R, \phi ; M, Q ; r]=S_{0}[\xi, R ; M, Q ; r]+\int \frac{Q}{c} \phi d r
$$

where $S_{0}$ is a functional that does not depend on $\phi$, while

$$
P_{\xi}=\frac{\delta S_{0}}{\delta \xi}, \quad P_{R}=\frac{\delta S_{0}}{\delta R} .
$$

The momentum $P_{\xi}$ (17) does not involve $\xi$ derivatives; therefore, the equation $P_{\xi}=\delta S_{0} / \delta \xi$ can be directly integrated [8] to yield

$$
\begin{gathered}
S_{0}[\xi, R ; M, Q ; r]=\int d r \int P_{\xi} d \xi+G[R ; M, Q ; r]= \\
=\frac{c^{3}}{\kappa} \int d r\left(\sqrt{\xi R F_{t o t}}-\frac{1}{2} R R_{, r} \ln \frac{R R_{, r}+\sqrt{\xi R F_{t o t}}}{R R_{, r}-\sqrt{\xi R F_{t o t}}}\right)+G[R ; M, Q ; r] .
\end{gathered}
$$

Here $G[R ; M, Q ; r]$ is a functional, which is independent of $\xi$. Taking into account (18), from (20) we obtain

$$
P_{R}=\frac{\delta S_{0}}{\delta R}=P_{R}+\frac{\delta}{\delta R} G[R ; M, Q ; r] .
$$

It follows that the integrability condition is satisfied if $G[R ; M, Q ; r]=G[M, Q ; r]$ is a functional, which is independent of the variable R. As a result, we come to the action functional

$$
S[\xi, R, \phi ; M, Q ; r]=\frac{c^{3}}{\kappa} \int d r\left(\sqrt{\xi R F_{t o t}}-\frac{1}{2} R R_{, r} \ln \frac{R R_{, r}+\sqrt{\xi R F_{t o t}}}{R R_{, r}-\sqrt{\xi R F_{\text {tot }}}}\right)+\int \frac{Q}{c} \phi d r+\int g d r
$$

in the $\mathrm{CS}$, as a solution of the EHJ equation. Here $g=g(M, Q ; r)$ is an arbitrary function of $M, Q$, and $r$. The motion trajectories follow from the equations

$$
\frac{\delta S}{\delta m}=0, \quad \frac{\delta S}{\delta Q}=0,
$$

which give as a result

$$
\begin{gathered}
\xi=R\left(\frac{f^{2}(r)}{c^{2}} F-\frac{R_{, r}^{2}}{F}\right), \quad \phi=\phi_{0}+\frac{f(r)}{c} \frac{Q}{R}, \\
F=-1+\frac{2 \kappa m}{c^{2} R}-\frac{\kappa Q^{2}}{c^{4} R^{2}}, \quad f=-\frac{\partial g(m, Q ; r)}{c \partial m}, \quad \phi_{0}=-c \frac{\partial(m, Q ; r)}{\partial Q} .
\end{gathered}
$$

The obtained relations determine the dependence of the dynamic variables $\xi$ and $\phi$ on the coordinate $r$, the variable $R(r)$ and its derivative with respect to $r$. For this solution, the metric (2) on $\mathrm{M}^{4}$ takes the form

$$
d s^{2}=\left(\frac{f^{2}(r)}{c^{2}} F-\frac{R_{, r}^{2}}{F}\right)^{-1}\left(n d x^{0}\right)^{2}-\left(\frac{f^{2}(r)}{c^{2}} F-\frac{R_{, r}^{2}}{F}\right)\left(d r+N^{r} d x^{0}\right)^{2}-R^{2} d \sigma^{2},
$$




$$
A=\frac{Q}{R} c d T=c \frac{Q}{R}\left(T_{, 0} d x^{0}+T_{, r} d r\right) .
$$

We determined $f(r)=c^{2} T_{, r}$, while $T_{, 0}$ is found from the integrability of the form $d T=T_{0} d x^{0}+T_{r} d r$. The function $n$ follows from the time recovery procedure.

\section{Configuration space metric}

It is obvious from the Lagrangian (4) that

$$
\frac{\partial \Lambda}{\partial n}=-\frac{T}{n^{2}}+U=0
$$

whence the relation

$$
n=\sqrt{U T}
$$

follows. Through excluding the function $n$ from the actions (4), it can be rewritten as follows

$$
S=\int \Lambda d^{2} x=2 \int \sqrt{U T} d^{2} x,
$$

or, taking into account the formula (5), in the form

$$
S=2 \int d r \int d x^{0} \sqrt{U\left[-\frac{c^{3}}{2 \kappa}\left(\xi_{, 0}-N^{r} \xi_{, r}-2 \xi_{N_{, r}}^{r}\right)\left(R_{, 0}-N^{r} R_{, r}\right)+\frac{1}{2 c} R^{2}\left(\phi_{, 0}-\varphi_{, r}\right)^{2}\right]} .
$$

We introduce the Lie differentials by the formulae:

$$
D \xi=d \xi_{, 0}-N^{r} \xi_{, r} d x^{0}-2 \xi N_{, r}^{r} d x^{0}, D R=d R-N^{r} R_{, r} d x^{0}, D \phi=d \phi-\varphi_{, r} d x^{0} .
$$

Then, the action $S$ takes the form

$$
S=2 \int d r \int D \Omega
$$

where

$$
D \Omega^{2}=U\left(D \Omega_{0}\right)^{2}=U\left(-\frac{c^{3}}{2 \kappa} D \xi D R+\frac{1}{2 c} D \phi^{2}\right)
$$

is the CS metric, $\left(D \Omega_{0}\right)^{2}$ is the minisuperspace metric, which is built on the kinetic part of (5). Note that in the T-region, when $R=c T\left(x^{0}\right)$ and $R_{, r}=R_{r r}=0$, then $U=c^{3} / 2 \kappa$ and both metrics coincide up to a coefficient. In the simplest case of the T-region, in the curvatures coordinates, when all spatial derivatives disappear, we have

$$
\left(D \Omega_{0}\right)^{2}=-\frac{c^{3}}{2 \kappa} d \xi d R+\frac{1}{2 c} d \phi^{2} .
$$

Then, using transformations of field variables:

$$
\xi=c \tau-x-\frac{y^{2}}{R}
$$




$$
\phi=\frac{c^{2}}{\sqrt{\kappa}} \frac{y}{R}, \quad R=c \tau+x,
$$

this metric is reduced to the Lorentzian form

$$
D \Omega^{2}=\frac{c^{3}}{2 \kappa}\left(D \Omega_{0}\right)^{2}=\frac{c^{2}}{4 \kappa}\left(-\frac{c^{4}}{\kappa} d \xi d R+d \phi^{2}\right)=\frac{c^{4}}{4 \kappa^{2}}\left(-c^{2} d \tau^{2}+d x^{2}+d y^{2}\right)
$$

\section{Conclusions}

The field's configuration under consideration admits two integrals of motion: the total mass and the charge, and therefore, the dynamical system turns out to be completely integrable. The mass function, together with the constraints and the charge, completely determines the expressions for the momenta in the CS in any reference frame. This allows you to find a common action in the CS, without solving the EGJ equation. Action derivatives with respect to parameters $M$ and $Q$ lead to trajectories of the system in the $\mathrm{CS}$, i.e. to the general solutions of Einstein equations in an implicit form.

We note that the minisuperspace metric, which is induced by the kinetic part of the Lagrangian, does not coincide with the CS metric arising when the factor $N$ is excluded from the action. In this paper, we introduce the metric $\mathrm{M}^{4}$ for which both supermetrics in the T-region coincide up to a coefficient. This simplifies subsequent calculations. In addition, one should take into account the fact that, when passing to the trivial foliation in the T-region, the CS is the flat and the minisupermetric admits the motions group $\mathrm{O}(1,2)$.

\section{References}

1. Gladush, V.D. On the configuration space of a spherically symmetric system of gravitational and electromagnetic fields / V.D. Gladush // Odessa Astronomical Publications. - 2018. - Vol. 31. - P. 15 - 19.

2. Gladush, V.D. On the quantization of black holes / V.D. Gladush // Vìsnik Dnìpropetropetrovs'kogo unìversitetu, Seria Fìzika, radioelectronika. - 2016. - Vol. 24, Issue 23, No. 2 - P. 31 - 34.

3. Kuchar, K.V. Geometrodynamics of Schwarzschild black holes / K.V. Kuchar // Phys. Rev. - 1994. - Vol. D50. - P. 3961 - 3981.

4. Louko, J. Hamiltonian thermodynamics of the Reissner-Nordström anti-de Sitter black hole / J. Louko, S. Winters-Hilt // Phys. Rev. D. - 1996. - Vol. 54, No. 4. - P. $2647-2663$.

5. Mäkelä. .I. Quantum-mechanical model of the Reissner-Nordström black hole / J. Mäkelä, P. Repo // Phys. Rev. D. - 1998. - Vol. 54, No. 2. - P. 4899 - 4916.

6. Gladush, V.D. Classical descriptions of the geometrodynamics of charged black holes / V.D. Gladush // Odessa Astronomical Publications. - 2019. - Vol. 32. - P. 35 39.

7. Gladush, V.D. Additional symmetry of spherically symmetric configurations, conservation laws and their application / V.D. Gladush, A.I. Petrusenko // Space, Time and Fundamental Interactions. - 2012. - No. 1. - P. 48 - 59 (in Russian).

8. Louis-Martinez, D. Exact Dirac quantization of all 2D dilaton gravity theories / D. Louis-Martinez, J. Gegenberg, G. Kunstatter // Physics Letters B. - 1994. - Vol. 321, No. 3. - P. 193 - 198. 